\title{
Influence of Configuration Mixing on Energies and Recombination Dynamics of Excitonic States in CdTe/ZnTe Quantum Dots
}

\author{
T. Smoleński ${ }^{a, *}$, T. KazimierczuK ${ }^{a}$, M. Goryca ${ }^{a, b}$, P. Kossacki ${ }^{a, b}$, J.A. GaJ $^{a}$,

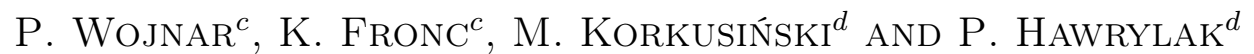 \\ ${ }^{a}$ Institute of Experimental Physics, Faculty of Physics, University of Warsaw, Hoża 69, 00-681 Warsaw, Poland \\ ${ }^{b}$ Grenoble High Magnetic Field Laboratory, CNRS, BP 166, 38042 Grenoble Cedex 9, France \\ ${ }^{c}$ Institute of Physics, Polish Academy of Sciences, al. Lotników 32/46, 02-668 Warsaw, Poland \\ ${ }^{d}$ Institute for Microstructural Sciences, National Research Council of Canada, Ottawa, K1A OR6, Canada \\ We study experimentally and theoretically excitonic recombination processes in $\mathrm{CdTe} / \mathrm{ZnTe}$ quantum dots. \\ The single quantum dot photoluminescence spectrum was observed and emission lines from $\mathrm{X}, \mathrm{X}^{-}, \mathrm{X}^{+}$and $2 \mathrm{X}$ \\ excitonic states were identified. Experimental results were analysed in the theoretical model based on the effective \\ mass approximation. Numerical calculations of energy positions and recombination probabilities of $\mathrm{X}, \mathrm{X}^{-}, \mathrm{X}^{+}$ \\ and $2 \mathrm{X}$ were performed. Computed results reproduce correctly the order and relative positions of emission lines \\ and ratios of radiative lifetimes.
}

PACS: 78.67.Hc, 78.55.Et

\section{Introduction}

Over many years semiconductor quantum dots (QD) have been extensively studied as a medium for storage and manipulation of quantum information [1,2]. Most experiments were performed on III-V structures due to their high structural quality. In comparison with classical GaAs-based III-V QD systems, II-VI QDs exhibit more robust excitonic states, therefore they emit light at higher temperatures. However, they still require more detailed studies, both theoretical and experimental. Interpretation of experimental results is often based on one electron picture. However, this picture is not sufficient for a quantitative description.

\section{Samples and experiment}

In this work we focus on the influence of configuration mixing on $\mathrm{CdTe} / \mathrm{ZnTe} \mathrm{QD}$ microphotoluminescence $(\mu \mathrm{PL})$ spectrum.

The experiments were carried out on samples containing self-assembled CdTe/ZnTe QDs. They were grown by molecular beam epitaxy on a GaAs substrate. The formation of the QDs was stimulated by deposition and subsequent desorption of an amorphous tellurium layer $[3,4]$.

For experimental studies of individual quantum dots, a $\mu \mathrm{PL}$ setup was used. The sample was fixed directly on

* corresponding author; e-mail: t.smolenski@student.uw.edu.pl the front surface of a mirror type microscope objective [5] and placed inside a cryostat at $T=1.6 \mathrm{~K}$. An argon ion laser $(\lambda=488 \mathrm{~nm})$ was used for non-resonant continuous wave excitation. For time-resolved measurements, frequency-doubled pulses of a mode-locked Ti:sapphire laser were used (repetition frequency of $76 \mathrm{MHz}$ ). The light emitted by the QDs was resolved in a monochromator equipped with a CCD camera and an avalanche photodiode (APD) serving as a single photon detector. For second order photon correlation measurements, a setup in Hunbury-Brown, Twiss configuration was used with two independently tunable detection arms [6].

A spectrally separated set of lines related to emission from a single $\mathrm{QD}$ was observed in the $\mu \mathrm{PL}$ spectrum (Fig. 1a). The identification of optical transitions in the $\mu \mathrm{PL}$ spectrum was based on a series of experiments, including measurements of $\mu \mathrm{PL}$ spectrum for different excitation powers, its polarization properties, and single photon correlation measurements performed in the Hanbury-Brown and Twiss setup. Obtained results were used to identify lines related to recombination of neutral exciton $(\mathrm{X})$, biexciton $(2 \mathrm{X})$ and charged excitons $\left(\mathrm{X}^{+}, \mathrm{X}^{-}\right)$. Relative energies and order of determined emission lines are consistent with previous experimental studies on $\mathrm{CdTe} / \mathrm{ZnTe}$ quantum dots [6]. The time-dependent intensities of $\mu \mathrm{PL}$ emission from $\mathrm{X}, 2 \mathrm{X}, \mathrm{X}^{-}$and $\mathrm{X}^{+}$states under pulsed excitation were also measured (Fig. 1b). Two components, a fast and a slow one, are present in all the recorded decays, in agreement with previous observations on CdTe/ZnTe QDs [7]. The data was fitted 
with monoexponential decay applied to the "fast" part of the decay curve. Obtained values of radiative lifetimes are equal to $\tau_{\mathrm{X}}=430 \pm 30 \mathrm{ps}, \tau_{2 \mathrm{X}}=270 \pm 30 \mathrm{ps}$, $\tau_{\mathrm{X}^{-}}=470 \pm 30 \mathrm{ps}$ and $\tau_{\mathrm{X}^{+}}=470 \pm 30 \mathrm{ps}$.

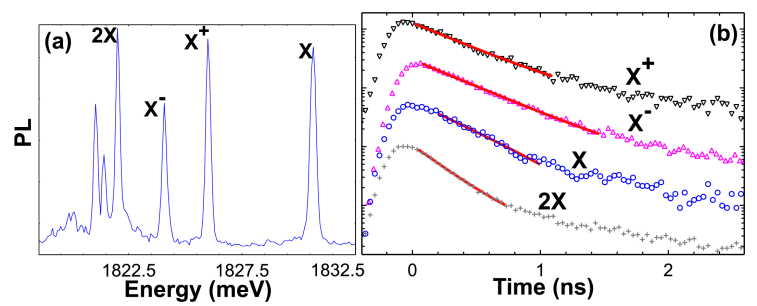

Fig. 1. (a) Typical photoluminescence spectrum of a single quantum dot. (b) Intensity of $\mathrm{X}, 2 \mathrm{X}, \mathrm{X}^{-}$and $\mathrm{X}^{+}$ $\mathrm{PL}$ as a function of time following excitation pulse. Solid lines represent exponential decays.

\section{Theoretical model}

We construct the theoretical model considering a single quantum dot in the effective mass approximation (EMA), following Hinzer et al. [8]. Due to the strain and confinement in the QDs, their light hole states are split off to higher energies and do not participate in the light emission. The single-particle basis from which the excitonic states are constructed consists therefore of heavy hole states with spin $z$-component $S_{\mathrm{h} z}= \pm 3 / 2$ and electron states with $S_{\mathrm{e} z}= \pm 1 / 2$. Typical lateral dimensions of self-assembled QDs are much larger than their thickness, so the considered problem can be treated as two-dimensional (2D). Following previous studies [9] we model the in-plane confinement of electrons and holes by a parabolic, symmetric potential $V(x, y)=m^{*} \omega^{2}\left(x^{2}+y^{2}\right) / 2$, where $m^{*}$ is the carrier effective mass and $\omega$ is its confinement frequency. The single particle states then correspond to the Fock-Darwin eigenstates $|n, m, s\rangle$, where $s$ is the spin $z$-component and $n, m$ are nonnegative integers related to angular momentum $\left(l_{z}|n, m, s\rangle=\hbar(n-m)|n, m, s\rangle\right)[9]$.

In order to obtain theoretical results for the exciton emission spectra, one has to consider many-particle states. With composite indices $i, j, k, l=(n, m)$, the Hamiltonian $H$ of the interacting electron-hole system may be written in the second quantization regime [10]:

$$
\begin{aligned}
H & =\sum_{i, \sigma} E_{i} c_{i, \sigma}^{\dagger} c_{i, \sigma}+\sum_{j, \tau} E_{j} h_{j, \tau}^{\dagger} h_{j, \tau} \\
& -\sum_{i, j, k, l, \sigma, \tau}\left\langle i, j\left|V_{\mathrm{eh}}\right| k, l\right\rangle c_{i, \sigma}^{\dagger} h_{j, \tau}^{\dagger} h_{k, \tau} c_{l, \sigma} \\
& +\frac{1}{2} \sum_{i, j, k, l, \sigma, \sigma^{\prime}}\left\langle i, j\left|V_{\mathrm{ee}}\right| k, l\right\rangle c_{i, \sigma}^{\dagger} c_{j, \sigma^{\prime}}^{\dagger} c_{k, \sigma^{\prime}} c_{l, \sigma} \\
& +\frac{1}{2} \sum_{i, j, k, l, \tau, \tau^{\prime}}\left\langle i, j\left|V_{\mathrm{hh}}\right| k, l\right\rangle h_{i, \tau}^{\dagger} h_{j, \tau^{\prime}}^{\dagger} h_{k, \tau^{\prime}} h_{l, \tau},
\end{aligned}
$$

where $\sigma, \tau$ are the spin $z$-components of the electron and hole, respectively. The operators $c_{i, s}^{\dagger}\left(c_{i, s}\right)$ and $h_{i, s}^{\dagger}\left(h_{i, s}\right)$ create (annihilate) the electron or hole in the state $|i, s\rangle$ with energy $E_{i}$. The first two terms in (1) describe the kinetic energy of electrons and holes, while the next three terms represent the Coulomb interaction between the carriers. The two-body matrix elements $\left\langle i, j\left|V_{a b}\right| k, l\right\rangle$ represent electron-electron $(a b=\mathrm{ee})$, hole-hole $(a b=\mathrm{hh})$ and electron-hole $(a b=\mathrm{eh})$ scattering, respectively [10, 11]. From (1) it can be seen that $H$ conserves the total spin $\boldsymbol{S}$ and its $z$-component $S_{z}$. Moreover, since electron-hole scattering does not change the $z$-component of angular momentum and total spin of the electron or hole system [10], the z-component of angular momentum and total spins $\boldsymbol{S}_{\mathrm{e}}$ and $\boldsymbol{S}_{\mathrm{h}}$ of electrons and holes are also conserved (we neglect electron-hole exchange interaction). Therefore, we can classify our states by $\boldsymbol{S}, S_{z}, l_{z}, \boldsymbol{S}_{\mathrm{e}}, \boldsymbol{S}_{\mathrm{h}}$. Furthermore, in order to find eigenstates of $H$, one can consider states with different values of defined quantum numbers separately. To describe excitonic recombination, we introduce the interband polarization operator $\mathcal{P}$ which annihilates the electron-hole pairs [10]:

$$
\mathcal{P}=\sum_{i, j}\left(c_{i, \uparrow} h_{j, \Downarrow}+c_{i, \downarrow} h_{j, \Uparrow}\right)\left\langle i \mid j_{\mathrm{R}}\right\rangle,
$$

where $j_{\mathrm{R}}=(m, n)$ for $j=(n, m)$. According to the $z$-component of photon angular momentum, only pairs with total spin \pm 1 can be annihilated. To calculate the emission spectrum we assume a fast energy relaxation inside the QD, which allows us to consider only emission from the ground level of each carrier configuration. We can express the relative probability $E\left(\omega, n_{\mathrm{e}}, n_{\mathrm{h}}, k\right)$ of recombination of the $|k\rangle$ ground state with single photon emission at angular frequency $\omega$ as

$$
E\left(\omega, n_{\mathrm{e}}, n_{\mathrm{h}}, k\right)=\sum_{f}|\langle f|\mathcal{P}| k\rangle|^{2} \delta\left(E_{k}-E_{f}-\hbar \omega\right),
$$

where $|f\rangle$ runs over all possible eigenstates of the final system, with one electron-hole pair less. Dirac delta in (3) determines possible photon energies, which correspond to the differences between initial and final state energies $E_{k}$ and $E_{f}$ for certain $f$. The term $|\langle f|\mathcal{P}| k\rangle|^{2}$ is equal to relative probability of an optical transition $|k\rangle \rightarrow|f\rangle$, which is directly proportional to $1 / \tau_{k f}$, where $\tau_{k f}$ is the radiative lifetime of state $|k\rangle$ related to $|k\rangle \rightarrow$ $|f\rangle$ transition. In this study we are not considering the excitation mechanisms, and therefore we focus on energies and recombination probabilities of the optical transitions. Numerical calculations of these probabilities can be much simplified within some additional assumptions on the values of confinement frequencies $\omega_{\mathrm{e}}, \omega_{\mathrm{h}}$. Previous studies, both theoretical [10] and experimental [12], revealed that for III-V QDs $m_{\mathrm{e}}^{*} \omega_{\mathrm{e}} \approx m_{\mathrm{h}}^{*} \omega_{\mathrm{h}}$. Our results show that the same approximation can be used also in case of $\mathrm{CdTe} / \mathrm{ZnTe} \mathrm{QDs}$. Therefore, electron and hole single particle envelope wave functions are almost identical, so we can use $\langle i \mid j\rangle=\delta_{i j}$ in (2). 


\section{Results and discussion}

In order to calculate emission spectra, we first have to know approximate values of the model parameters. Electron and hole effective masses in CdTe are given by $m_{\mathrm{e}}^{*}=0.1 m_{0}$ and $m_{\mathrm{h}}^{*}=0.2 m_{0}\left(m_{0}\right.$ is the free electron mass) [13], while $\epsilon_{r}=10.2$ [14]. The confinement frequencies $\omega_{\mathrm{e}}$ and $\omega_{\mathrm{h}}$ have to be fitted to the experimental data. In the first approximation we can assume, that energetic distance between $s$ and $p$ shell emission observed in $\mathrm{QD} \mu \mathrm{PL}$ spectra [15] is equal to the sum of $E_{10}-E_{00}$ for electrons and holes. This analysis yields $\hbar\left(\omega_{\mathrm{e}}+\omega_{\mathrm{h}}\right)=50 \mathrm{meV}$. In order to find $\omega_{\mathrm{e}}$ and $\omega_{\mathrm{h}}$ separately, one can calculate the positions of excitonic emission lines and compare them with the experimental data. We focus on the $\mathrm{X}, \mathrm{X}^{-}, \mathrm{X}^{+}$and $2 \mathrm{X}$ states identified in the spectrum in Fig. 1b. Theoretical analysis shows that each of these states has one bright emission line in its spectrum. Their relative energetic positions were calculated numerically for $\hbar \omega_{\mathrm{e}} \in[0,50] \mathrm{meV}$. Hamiltonian diagonalization was performed in the basis containing states with carriers on the $s$ and $p$ shells [9]. The results are presented in Fig. 2a. It is easy to notice that for small values of $\omega_{\mathrm{e}}$ the $\mathrm{X}^{-}$emission line has the lowest energy. When $\omega_{\mathrm{e}}$ grows, hole confinement frequency $\omega_{\mathrm{h}}$ becomes smaller and $\mathrm{X}^{+}$emission line energy becomes the lowest. The correct order of emission lines, where $2 \mathrm{X}$ line has the lowest energy, can be reproduced only for $\hbar \omega_{\mathrm{e}}$ in a very small range near $33.5 \mathrm{meV}$ value, for which $m_{\mathrm{e}}^{*} \omega_{\mathrm{e}} \approx m_{\mathrm{h}}^{*} \omega_{\mathrm{h}}$. For further calculations we will assume that $\hbar \omega_{\mathrm{e}}=32.5 \mathrm{meV}$ and $\hbar \omega_{\mathrm{h}}=17.5 \mathrm{meV}$.

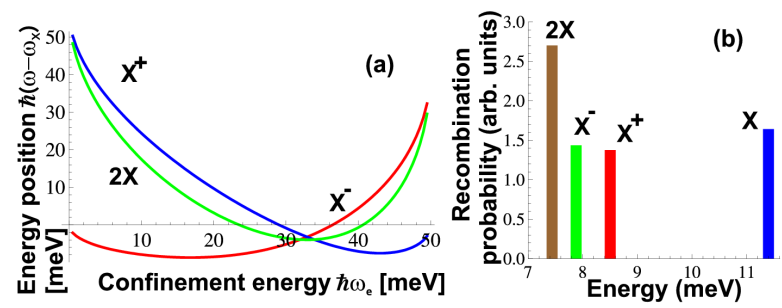

Fig. 2. (a) Energy positions of $\mathrm{X}^{-}, \mathrm{X}^{+}$and $2 \mathrm{X}$ emission lines $\hbar \omega$ relative to the energy $\hbar \omega_{\mathrm{X}}$ of $\mathrm{X}$ line as a function of electron confinement energy $\hbar \omega_{\mathrm{e}}$ for $\hbar\left(\omega_{\mathrm{e}}+\omega_{\mathrm{h}}\right)=50 \mathrm{meV}$. (b) Histogram of energy positions of $\mathrm{X}, \mathrm{X}^{-}, \mathrm{X}^{+}$and $2 \mathrm{X}$ emission lines. Line heights are directly proportional to recombination probabilities.

Knowing all necessary parameters we calculated numerically the energy positions and recombination probabilities of X, $\mathrm{X}^{-}, \mathrm{X}^{+}$and $2 \mathrm{X}$ excitonic states. Obtained results are presented in Fig. 2b. Comparing them with the experimental spectrum in Fig. 1a, we see that our model reproduces correctly the order and relative positions of considered emission lines. Moreover, line heights in Fig. $2 \mathrm{~b}$ are directly proportional to inverse recombination time of each state, so we can calculate the ratios of radiative lifetimes. Their values are $\tau_{\mathrm{X}} / \tau_{\mathrm{X}^{-}}=0.87$, $\tau_{\mathrm{X}} / \tau_{\mathrm{X}^{+}}=0.84, \tau_{\mathrm{X}} / \tau_{2 \mathrm{X}}=1.64$, remaining in excellent agreement with experimentally obtained $\tau_{\mathrm{X}} / \tau_{\mathrm{X}^{-}}=0.9$,
$\tau_{\mathrm{X}} / \tau_{\mathrm{X}^{+}}=0.9$ and $\tau_{\mathrm{X}} / \tau_{2 \mathrm{X}}=1.6$. A check of the final results was performed by repeating the calculations for the same parameters in an extended basis including $s$, $p$, and $d$ shells. The agreement of the results with the experimental data remained the same.

\section{Conclusion}

Summarizing, time-resolved $\mu \mathrm{PL}$ spectra of individual $\mathrm{CdTe} / \mathrm{ZnTe} \mathrm{QDs}$ were measured. The energetic positions of emission lines and relative radiative lifetimes were determined experimentally and calculated for $\mathrm{X}, \mathrm{X}^{-}, \mathrm{X}^{+}$ and $2 \mathrm{X}$ excitonic states. The comparison with experimental data revealed that $\mu \mathrm{PL}$ spectrum of a single $\mathrm{CdTe} / \mathrm{ZnTe} \mathrm{QD}$ can be correctly reproduced by introduced theoretical model. In the future, the model can be applied for higher excitonic states and hopefully help in identification of emission lines in the $p$-shell emission.

\section{Acknowledgments}

This work was partially supported by the Polish Ministry of Science and Higher Education as research grants in years 2008-2011, by European Project No. MTKD-CT-2005-029671 and Foundation for Polish Science.

\section{References}

[1] P. Hawrylak, M. Korkusiński, Topics Appl. Phys. 90, 25 (2003).

[2] P. Michler, Single Semiconductor Quantum Dots, Springer-Verlag, Berlin 2009.

[3] F. Tinjod, S. Moehl, K. Kheng, B. Gelles, H. Mariette, Appl. Phys. Lett. 82, 4340 (2003).

[4] P. Wojnar, , J. Suffczyński, K. Kowalik, A. Golnik, M. Aleszkiewicz, G. Karczewski, J. Kossut, Nanotechnology 19, 235403 (2008).

[5] J. Jasny, J. Sepiol, T. Imgartinger, M. Trauber, A. Renn, U.P. Wild, Rev. Sci. Instrum. 67, 1425 (1996).

[6] J. Suffczynski, T. Kazimierczuk, M. Goryca, B. Piechal, A. Trajnerowicz, K. Kowalik, P. Kossacki, A. Golnik, K.P. Korona, M. Nawrocki, J.A. Gaj, Phys. Rev. B 74, 085319 (2006).

[7] B. Piechal, J. Suffczyński, A. Trajnerowicz, M. Goryca, P. Kossacki, A. Golnik, J.A. Gaj, Acta Phys. Pol. A 108, 831 (2005).

[8] K. Hinzer, P. Hawrylak, M. Korkusinski, S. Fafard, M. Bayer, O. Stern, A. Gorbunov, A. Forchel, Phys. Rev. B 63, 75314 (2001).

[9] P. Hawrylak, Phys. Rev. B 60, 5597 (1999).

[10] L. Jacak, P. Hawrylak, A. Wójs, Quantum Dots, Springer-Verlag, Berlin 1998.

[11] A. Wójs, P. Hawrylak, Phys. Rev. B 51, 880 (1995).

[12] S. Raymond, S. Fafard, P. J. Poole, A. Wojs, P. Hawrylak, S. Charbonneau, D. Leonard, R. Leon, P. M. Petroff, J. L. Merz, Phys. Rev. B 54, 11548 (1996).

[13] G. Fishman, Phys. Rev. B 52, 11132 (1995).

[14] Landolt-Börnstein - Group III Condensed Master, Springer-Verlag, Berlin 1987.

[15] D. Chithrani, M. Korkusinski, S.J. Cheng, P. Hawrylak, R.L. Williams, J. Lefebvre, P.J. Poole, G.C. Aers, Physica E 26, 322 (2005). 